\title{
Dense array EEG source estimation in neocortical epilepsy
}

\author{
Madoka Yamazaki ${ }^{1,2}$ *, Don M. Tucker ${ }^{3,4}$, Marie Terrill ${ }^{4}$, Ayataka Fujimoto ${ }^{2}$ and Takamichi Yamamoto ${ }^{2}$ \\ 1 Department of Health Science, Daito Bunka University, Saitama, Japan \\ ${ }^{2}$ Comprehensive Epilepsy Center, Seirei Hamamatsu General Hospital, Shizuoka, Japan \\ ${ }^{3}$ Department of Psychology, University of Oregon, Eugene, OR, USA \\ ${ }^{4}$ Electrical Geodesics, Inc., Eugene, OR, USA
}

\section{Edited by:}

Mark Holmes, University of

Washington, USA

\section{Reviewed by:}

Mario A. Vanegas, Instituto Nacional de Neurologia y Neurocirugia, Mexico Erik K. St. Louis, Mayo Clinic and

Foundation, USA

*Correspondence:

Madoka Yamazaki, Department of Health Science, Daito Bunka

University, 560 Iwadono,

Higashimatsuyama, Saitama,

430-8558, Japan.

e-mail: madokaymzk@gmail.com
Rationale: Dense array EEG (dEEG) evenly covers the whole head surface with over 100 channels contributing to more accurate electrical source imaging due to the higher spatial and temporal resolution. Several studies have shown the clinical utility of dEEG in presurgical clinical evaluation of epilepsy. However validation studies measuring the accuracy of dEEG source imaging are still needed. This can be achieved through simultaneously recording both scalp dEEG with intracranial electrodes (icEEG), which is considered as the true measure of cortical activity at the source. The purpose of this study is to evaluate the accuracy of 256-channel dEEG electrical source estimation for interictal spikes.

Methods: Four patients with medically refractory neocortical epilepsy, all surgical candidates, underwent subdural electrode implantation to determine ictal onset and define functional areas. One patient showed a lesion on the magnetic resonance imaging in the right parietal lobe. The patient underwent simultaneous recording of interictal spikes by both scalp 256-channelsvdEEG and icEEG. The dEEG was used to non-invasively estimate the source of the interictal spikes detected by the 256-channel dEEG array, which was then compared to the activity measured directly at the source by the icEEG.

Results: From the four patients, a total of 287 interictal spikes were measured with the icEEG. One hundred fifty-five of the 287 spikes $(54 \%)$ were visually detected by the dEEG upon examination of the 256 channel head surface array. The spike amplitudes detected by the 256-channel dEEG correlated with icEEG spike amplitudes $(p<0.01)$. All spikes detected in dEEG were localized to the same lobe correctly.

Conclusion: Our study demonstrates that 256-channel dEEG can reliably detect interictal spikes and localize them with reasonable accuracy. Two hundred fifty-six-channel dEEG may be clinically useful in the presurgical workup for epilepsy and also reduce the need for invasive EEG evaluation.

Keywords: dense array EEG, source estimation, neocortical epilepsy, interictal spike, intracranial EEG

\section{INTRODUCTION}

For patients with intractable epilepsy, surgical therapy is an important treatment option. Although the propagation of seizures may involve complex cerebral networks, surgical resection of the cortical zone of seizure onset may be effective, if this zone can be identified and delineated from surrounding tissue. The first stage of diagnostic tests is typically non-invasive, with the goal being to characterize the epileptogenic zone as comprehensively as possible in order to guide the next stage of invasive testing with intracranial electrodes that confirms the decision on resective surgery.

Magnetoencephalography (MEG) and electroencephalography (EEG) have both been used as non-invasive methods in the electrophysiological evaluation of the epileptogenic zone. A number of studies has shown that multi-channel MEG is useful for dipole localization of interictal spike events, especially in neocortical epilepsy, where the sources may be more superficial (closer to the skull) compared to epilepsy with deeper sources, such as mesial temporal lobe epilepsy (Ricci et al., 1987; Gallen et al., 1995;
Knowlton et al., 1997; Stefan et al., 2003; Huiskamp et al., 2010). MEG is inherently insensitive to deeper brain sources because magnetic signals fall off by the square of the distance from the source. Although EEG has better depth sensitivity than MEG, and it is sensitive to radial (gyral) as well as tangential (sulcal) sources, the electrical volume conduction of EEG is distorted by the resistive skull, requiring detailed computational modeling for an accurate inverse estimation (Ebersole, 2000; Vanrumste et al., 2000; Baumgartner, 2004; Plummer et al., 2007).

Although accurate electrical head modeling is now available for EEG, based on the patient's magnetic resonance imaging (MRI) and CT (for skull conductivity), the conventional 21channel scalp EEG does not have high enough spatial resolution to allow for accurate source localization in most cases (Tucker et al., 2004; Holmes et al., 2005). Adequate source localization requires adequate spatial sampling in addition to sophisticated source analysis techniques. Freeman et al. (2003) reported that the spatial Nyquist of the human scalp EEG necessitates 
inter-sensor distances of $<10 \mathrm{~mm}$. Recently it has become possible to record dense array EEG (dEEG) of up to 256 channels, providing 20-25 mm interelectrode distances for most adult head sizes. Several studies have shown that interictal source localization with dEEG suggests its usefulness in neurosurgical planning in epilepsy (Lantz et al., 2003a,b; Michel et al., 2004a; Holmes, 2008). However validation studies measuring the accuracy of dEEG source imaging are still needed. This can be achieved through simultaneously recording the scalp dEEG with intracranial electrodes (icEEG), which provides a local measure of electrical activity at the gyral cortical (pial) surface. In previous studies we have investigated the sensitivity and accuracy of dEEG source analysis comparing 256-channel dEEG source analysis of interictal activity and simultaneously recorded icEEG in patients with mesial temporal lobe epilepsy (Yamazaki et al., $2012 a, b)$. In the present study, we extend this approach to investigate the accuracy of source estimation with dEEG in patients with neocortical epilepsy using simultaneous dEEG and icEEG recording.

\section{MATERIALS AND METHODS PATIENTS}

We studied four patients, each of whom had suffered from intractable localization related epilepsy for at least 2 years. Each patient was a surgical candidate and underwent a presurgical workup including conventional long-term EEG monitoring, MRI, 125-Iomazenil (IMZ)-single-photon emission tomography (SPECT), 18-F fluorodeoxyglucose (FDG)-Positron emission tomography (PET), and neuropsychological testing. Clinical information for these patients is summarized in Table 1. Case 1 had right parietal cortical dysplasia.

We received approval for this study from Seirei Hamamatsu General Hospital Human Subject Committee and informed consent was obtained from all patients.

\section{INTRACRANIAL EEG RECORDING}

Subdural strip and grid electrodes (Unique medical, Tokyo, Japan) were implanted for each patient in order to delineate the epileptogenic zone for cortical excision, and to separate it from functional areas. All electrode contacts were platinum, and interelectrode distance was $10 \mathrm{~mm}$. The location and types of subdural electrodes used for each patient are shown in Table 1 .

\section{DENSE ARRAY EEG RECORDING}

The dEEG was recorded with the 256-channel Geodesic Sensor Net (Electrical Geodesics, Inc., Eugene, OR, USA), providing 257 electrodes (including vertex reference) covering the face and neck as well as cranium, with $20-25 \mathrm{~mm}$ interelectrode distances. The coverage of the face and neck is important for measuring the downward projection of electrical potentials from basal brain regions.

\section{SIMULTANEOUS icEEG AND dEEG ACQUISITION}

We conducted simultaneous icEEG recording with NicoletOne (CareFusion, Middleton, WI, USA) and dEEG with Net Amp300 (Electrical Geodesics, Inc., Eugene, OR, USA) at $1 \mathrm{kHz}$ sampling with bandpass filter 0.1 and $400 \mathrm{~Hz}$ for approximately 30-40 min. A digital pulse from the icEEG system was provided to the dEEG acquisition system for synchronization. Prior to the simultaneous icEEG-dEEG recording, each patient recovered from the intracranial placement for at least 3 days, allowing surgical wounds to heal to avoid infection risk during simultaneous scalp recording. There were no complications due to any of the simultaneous recordings.

\section{DATA ANALYSIS}

Frequent epileptiform discharges were selected during artifact free periods in the simultaneous dEEG and icEEG recordings. Interictal spikes seen in icEEG were visually identified by a certified

Table 1 | Case summary.

\begin{tabular}{|c|c|c|c|c|}
\hline & Case 1 & Case 2 & Case 3 & Case 4 \\
\hline Age/sex & 28 year/F & 38 year/M & 27 year/M & 21 year/F \\
\hline Onset & 3 year & 8 year & 5 year & 17 year \\
\hline $\mathrm{MRI}$ & R-P cortical dysplasia & Normal & Normal & Normal \\
\hline IMZ-SPECT* ${ }^{*}$ & $\mathrm{R}-\mathrm{P}$ & L-F, mT, laT & Bilateral-F, P & $\mathrm{R} \mathrm{mT}$ \\
\hline Interictal & C4, P4 & Fp1, F7, T3 & $\mathrm{C} 4, \mathrm{P} 4, \mathrm{~T} 6$ & $\mathrm{Fp} 2, \mathrm{~F} 8, \mathrm{~T} 4$ \\
\hline $\begin{array}{l}\text { Subdural electrodes } \\
\text { location }\end{array}$ & & & & \\
\hline
\end{tabular}

SPS, simple partial seizure; CPS, complex partial seizure; sGTC, secondary generalized tonic clonic seizure; ", hypoperfusion area; ${ }^{* *}$, hypometabolism area; $m T$, mesial temporal lobe; laT, lateral temporal lobe. 
EEG technologist (MY) and confirmed by a board-certified clinical epileptologist (AF). As expected, many of the icEEG spikes were not detectible by inspection of the dEEG. We calculated the spike detection rate of 256-channel dEEG (with the icEEG detection as the criterion), and we measured the average maximum amplitude for both the icEEG detected spikes and the smaller subset of dEEG detected spikes.

Electrical source localization was conducted at the rising phase for the dEEG detected spikes with a linear inverse method (LAURA) using the GeoSource 1.0 software package ${ }^{1}$ within the space of a 3D head model derived from the Montreal Neurological Institute's average adult MRI. The LAURA constraint provides results very similar to the low-resolution electromagnetic tomography (LORETA) spatial Laplacian constraint (Pascual-Marqui et al., 2002), and this smoothing constraint has been shown to provide stable source estimation of interictal epileptiform events in neurosurgical planning for epilepsy (Lantz et al., 2003a,b; Michel et al., 2004a,b).

\section{RESULTS}

\section{SPIKE DETECTION RATE}

A total of 287 spikes were recorded in icEEG with four patients during recordings of approximately $30-40 \mathrm{~min}$ each. One hundred

${ }^{1}$ http://www.egi.com fifty-five of these spikes (54\%) were also clearly distinguishable from background activity in the simultaneously recorded dEEG. The dEEG detection rate for the spikes verified by icEEG for each patient is summarized in Table 2. Case 2 showed two independent patterns of interictal spikes, one originating from the left frontal region and the other from the left mesial temporal region. The detection rate of neocortical spikes was $56 \%$ and that for mesial temporal spikes in this case was $39 \%$.

\section{icEEG AMPLITUDE OF DETECTABLE SPIKES}

The average maximal icEEG amplitude of neocortical interictal spikes that were also detectable by the scalp dEEG was $968 \mu \mathrm{V}$ (standard deviation of $139 \mu \mathrm{V})$, significantly higher $(p<0.01)$ than that of dEEG undetectable spikes $(757 \mu \mathrm{V}$; standard deviation of $102 \mu \mathrm{V})$. The average maximal icEEG amplitude of mesial temporal spikes that were also detectable by the scalp dEEG was $1112 \mu \mathrm{V}$ (standard deviation of $197 \mu \mathrm{V}$ ), significantly higher $(p<0.01)$ than that of dEEG undetectable spikes $(763 \mu \mathrm{V}$; standard deviation of $172 \mu \mathrm{V})$.

\section{DENSE ARRAY EEG SOURCE ESTIMATION}

Figure 1 shows dEEG source estimation for the dEEG detected icEEG spikes in each patient, visualized by displaying the voxel with maximal source amplitude (as well as voxels with similar

Table 2 | Spike detection rate.

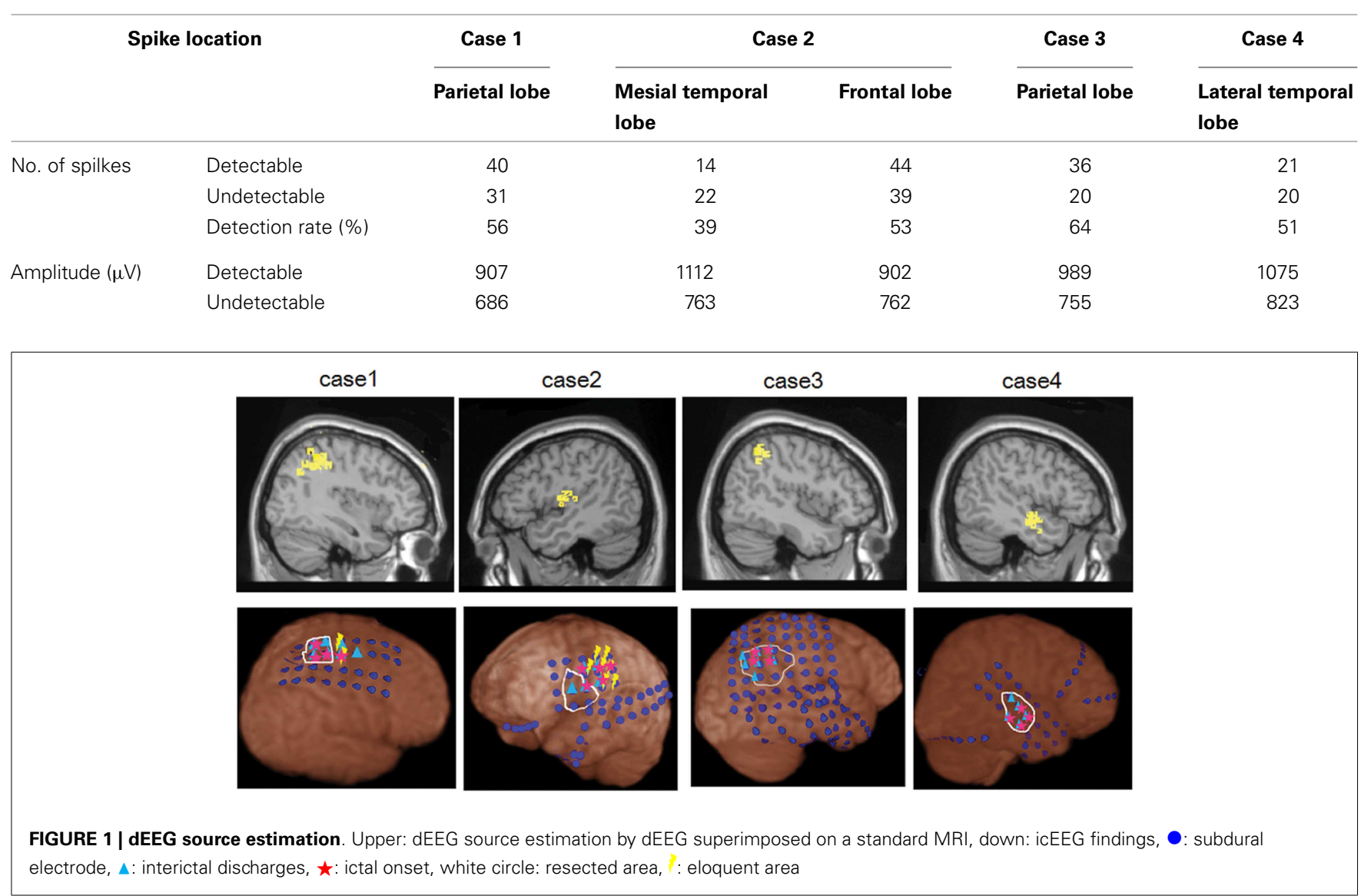


amplitudes) using a section from the MNI typical brain image ${ }^{2}$ consistent with the MNI atlas used for the cortical dipole locations in the GeoSource software. All spikes detected in dEEG were localized to the same lobe correctly and 141 of 155 spikes (91\%) were well localized, close to the position confirmed by subdural electrodes.

Figure 2 shows more detail for a typical example of icEEG and dEEG source estimation. For this selected spike, the icEEG and conventional EEG recordings are shown in Figure 2A. The locations of the icEEG strips are shown in Figure 2B. The head surface (scalp, face, and neck) distribution of the dEEG potentials is shown in Figure 2C. From inspection, this dEEG head surface topography is consistent with a mostly radial source in the right posterior midline, with diffuse inversions over both sides of the face indicating the approximate radial source orientation. The peak of the non-invasive dEEG source estimation for this spike is shown in Figure 2D consistent with visual inspection of the surface array, and the post-operative MRI shows the resected region in Figure 2E.

\section{SURGICAL OUTCOME}

All patients underwent resective surgery based on the intracranial icEEG findings, functional mapping, and imaging studies. Pathological findings are case 1: FCD type IIB, case 2: FCD type IIB, case 3: FCD type IA, and case 4: no abnormality. Surgical outcome is Engel class Ia (case 3,4), IId (case 1), and IIIa (case 2) within the post-operative follow-up period 23-35 months at this time (mean, 30 months).

\section{DISCUSSION}

Simultaneous recording of non-invasive dEEG with icEEG allowed a direct comparison of the sensitivity of dEEG to epileptiform events (spikes) that were confirmed by intracranial recordings. In these four patients with neocortical (extra temporal) epilepsy, the non-invasive dEEG localization of spikes predicted not only the intracranial location of the spikes, but also the cortical location of seizure onset (Figure 1).

Clearly there were many spikes visible in the icEEG that were not detected through visual inspection of the dEEG. For the neocortical spikes identified by icEEG in the present study, the dEEG spike detection rate was $56 \%$. As would be expected, the dEEG detected spikes were consistently larger in amplitude than the dEEG undetected spikes in each patient. For the mesial temporal spikes examined in our previous studies with simultaneous icEEG and dEEG (Yamazaki et al., 2012a,b), the dEEG detection rates were somewhat lower, 45 and $42 \%$, respectively. Also as expected, to be detectable by visual inspection of the dEEG, the mesial temporal lobe spikes in those studies were typically larger in amplitude (averaging $1236 \mu \mathrm{V}$; Yamazaki et al., 2012b) compared with the dEEG detected neocortical spikes of the present study (averaging $968 \mu \mathrm{V}$ ). For the one patient of the present study with mesial temporal spikes, the 39\% dEEG detection rate was similar to the previous rates for detecting mesial temporal spikes.

\footnotetext{
${ }^{2}$ www.bic.mni.mcgill.ca
}

A higher non-invasive detection sensitivity to neocortical than mesial temporal spikes has also been observed in those MEG studies where validation has also been obtained from simultaneous icEEG recording. Compared with dEEG, MEG is particularly insensitive to the deep sources presented by mesial temporal lobe spikes. Huiskamp et al., 2010 reported that whole head MEG detected only $28 \%$ of the icEEG spikes generated in the mesial temporal lobe, whereas it detected $70 \%$ of the icEEG detected spikes from the lateral temporal lobe and extra temporal regions. Similarly, Oishi et al., 2002 observed that MEG detected only $26 \%$ of the mesial temporal spikes, but $53 \%$ of lateral frontal spikes that were confirmed by simultaneous icEEG recording. Considering our previous results and the present study, 256-channel dEEG is roughly equivalent to whole head MEG in detecting neocortical sites but superior to MEG in detecting mesial temporal lobe spikes.

Magnetic fields are less distorted by the skull, cerebral fluid, and scalp than electrical fields (Nakasato et al., 1994; Ebersole, 1999; Minassian et al., 1999; Otsubo et al., 1999; Morioka et al., 2000). However, the accuracy of MEG for deep sources, such as in the mesial temporal lobe, appears limited because magnetic signals fall off by the square of the distance (Mikuni et al., 1997; Oishi et al., 2002; Rampp and Stefan, 2007). In addition, the 256channel dEEG sensor net includes sensors on the face and neck that improve the characterization of the electrical fields from the temporal lobe that are directed inferiorly and anteriorly, where even whole head MEG has limited coverage. Finally, the inclusion of accurate head conductivity information, such as from the MRI and CT atlas used for finite difference conductivity modeling in GeoSource software in the present analyses, is now helping to deal with the ambiguities of electrical volume conduction that plague EEG source localization.

Presurgical evaluation for what is typically called neocortical epilepsy, located outside the mesial temporal lobe (and archicortical hippocampus), requires precise accuracy because the epileptogenic zones are often located adjacent to critical functional areas of cortex (Otsubo et al., 1997; Minassian et al., 1999; Chitoku et al., 2001). For the four patients of the present study, the noninvasive dEEG source localization of the patient's typical interictal spikes was predictive of the icEEG localization of not only the interictal spikes but also the seizure onset. Although the interictal events do not always localize to the same region as seizure onset, the utility of careful non-invasive localization of interictal events as a first stage in the presurgical workup is consistent with previous dEEG studies on electric source imaging in epilepsy (Michel et al., 2004b; Brodbeck et al., 2010). Nonetheless, noninvasive localization of seizure onset is an important confirmation for the hypotheses generated from localizing interictal events, and may be an important form of converging evidence in preparing for icEEG mapping of seizure onset (Holmes et al., 2008, 2010). Non-invasive 256-channel dEEG is now available for long-term monitoring for seizure onset; although more challenging than conventional EEG monitoring, it is more practical than attempting seizure monitoring with MEG.

There were several important technical limitations of this study that should be addressed in future research. We used standard $3 \mathrm{D}$ sensor positions, rather than the precise 3D locations of the 256-channels for each individual net application, such as can be 


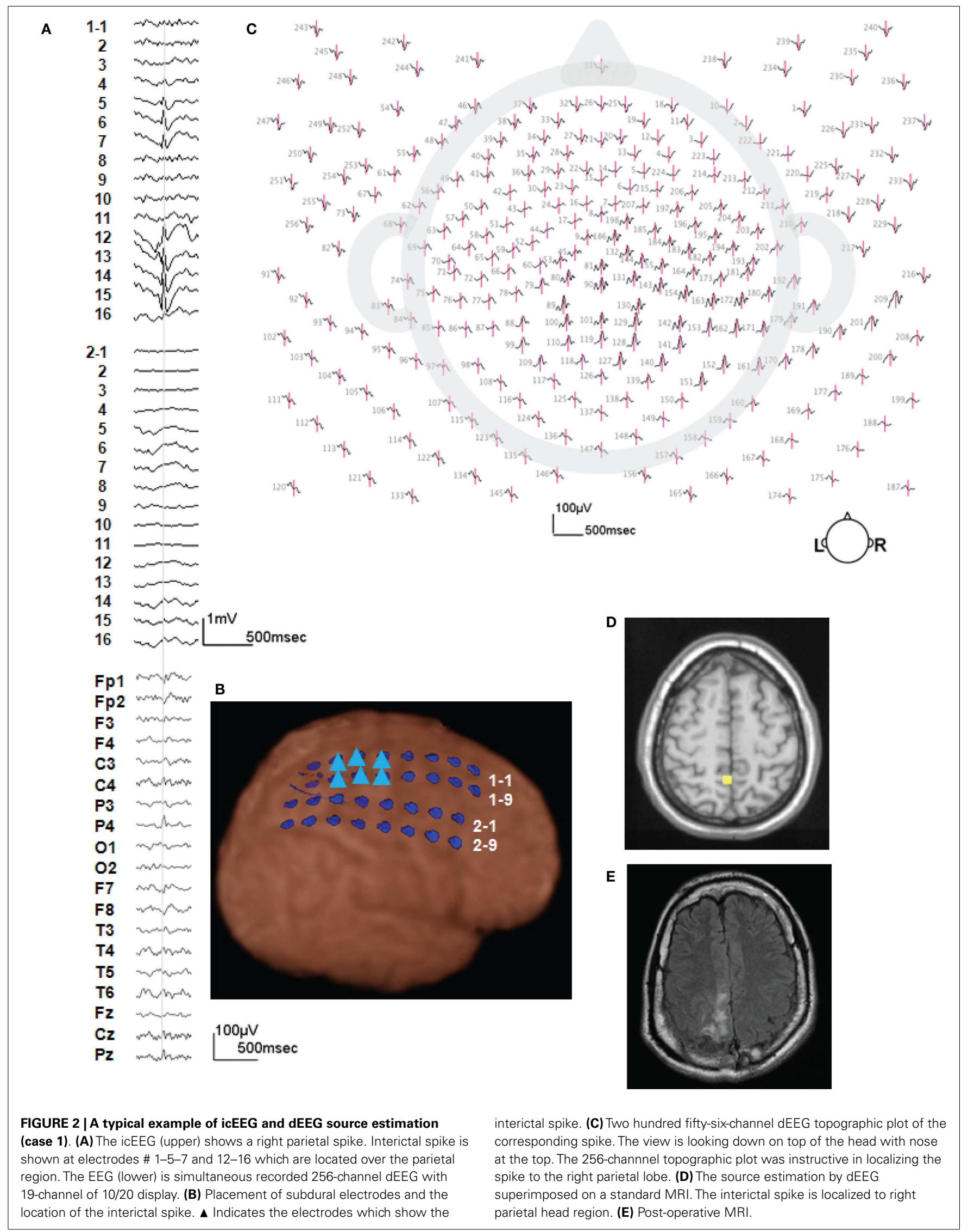


obtained with photogrammetry (Russell et al., 2005). Even with careful placement of the net, the sensor positions can vary in relation to the cortex from patient to patient depending on head shape and the tissues of the face and neck. In addition, we used the GeoSource atlas finite difference model of head conductivity, based on the MNI brain template (and skull CT fit to that template), rather than building an electrical conductivity model based on the individual's MRI and CT for the source estimation. Brodbeck et al., 2011 examined the sensitivity and specificity of dEEG source estimation comparing individual MRI and standard MRI template and showed the benefit of using individual MRI. For example, Case 1 in the present series had a focal cortical dysplasia in the right parietal region; rather than using the GeoSource atlas it would have been useful if we could compare

\section{REFERENCES}

Baumgartner, C. (2004). Controversies in clinical neurophysiology. MEG is superior to EEG in the localization of interictal epileptiform activity: Con. Clin. Neurophysiol. 115, 1010-1020.

Brodbeck, V., Spinelli, L., Lascano, A. M., Pollo, C., Schaller, K., Vargas, M. I., et al. (2010). Electrical source imaging for presurgical focus localization in epilepsy patients with normal MRI. Epilepsia 51, 583-591.

Brodbeck, V., Spinelli, L., Lascano, A. M., Wissmeier, M., Vargas, M. I., Vulliemoz, S., et al. (2011). Electroencephalographic source imaging: a prospective study of 152 operated epileptic patients. Brain 34(Pt 10), 2887-2897.

Chitoku, S., Otsubo, H., Harada, Y., Jay, V., Rutka, J. T., Weiss, S. K., et al. (2001). Extraoperative cortical stimulation of motor function in children. Pediatr. Neurol. 24, 344-350.

Ebersole, J. S. (1999). Non-invasive presurgical evaluation with EEG/MEG source analysis. Electroencephalogr. Clin. Neurophysiol. 50, 167-174.

Ebersole, J. S. (2000). Noninvasive localization of epileptogenic foci by EEG source modeling. Epilepsia 41(Suppl. 3), 24-33.

Freeman, W., Holmes, M. D., Burke, B., and Vanhatalo, S. (2003). Spatial spectra of scalp EEG and EMG from awake humans. Clin. Neurophysiol. 114, 1053-1069.

Gallen, C. C., Hirschkoff, E. C., and Buchanan, D. S. (1995). Magnetoencephalography and magnetic source imaging. Capabilities and limitations. Neuroimaging Clin. N. Am. 5, 227-249.

Holmes, M. D. (2008). Dense array EEG: methodology and new hypothesis on epilepsy syndromes. Epilepsia 49(Suppl. 3), 3-14.
Holmes, M. D., Brown, M., and Tucker, D. M. (2005). Dense array EEG and source analysis reveal spatiotemporal dynamics of epileptiform discharges. Epilepsia 46(Suppl. 8), 136.

Holmes, M. D., Brown, M., Tucker, D. M., Saneto, R. P., Miller, K. J., Wig, G. S., et al. (2008). Localization of extratemporal seizure with noninvasive dense-array EEG. Pediatr. Neurosurg. 44, 474-479.

Holmes, M. D., Tucker, D. M., Quiring, J. M., Hakimian, S., Miller, J. W., and Ojemann, J. G. (2010). Comparing noninvasive dense array and intracranial electroencephalography for localization of seizures. Neurosurgery 66, 354-362.

Huiskamp, G., Agirre-Arrizubieta, Z., and Leijten, F. (2010). Regional differences in the sensitivity of MEG for interictal spikes in epilepsy. Brain Topogr. 23, 159-164.

Knowlton, R. C., Laxer, K. D., Aminoff, M. J., Roberts, T. P., Wong, S. T., and Rowley, H. A. (1997). Magnetoencephalography in partial epilepsy: clinical yield and localization accuracy. Ann. Neurol. 42, 622-631.

Lantz, G., Grave de Peralta, R., Spinelli, L., Seeck, M., and Michel, C. M. (2003a). Epileptic source localization with high density EEG: how many electrodes are needed? Clin. Neurophysiol. 114, 63-69.

Lantz, G., Spinelli, L., Seeck, M., de Peralta Menendez, R. G., Sottas, C. C., and Michel, C. (2003b). Propagation of interictalepileptiform activity can lead to erroneous source localizations: a 128-channel EEG mapping study. J. Clin. Neurophysiol. 20, 311-319.

Michel, C. M., Lantz, G., Spinelli, L., De Peralta, R. G., Landis, T., and Seeck, M. (2004a). 128-Channel EEG source imaging in epilepsy:

the source localization of the spikes with the lesion location precisely by building the conductivity model from the patient's MR and CT.

\section{CONCLUSION}

Simultaneous recording of dEEG and icEEG allows direct examination of the accuracy of localizing cortical electrical sources from non-invasive dEEG recordings. In four patients with neocortical epilepsy examined with 256-channel dEEG, we observed good prediction of both the interictal spikes and seizure onset as verified by icEEG. Careful non-invasive analysis of the patient's typical interictal events with dEEG may be an important first step in the neurosurgical planning for resection of the seizure onset zone.

clinical yield and localization precision. J. Clin. Neurophysiol. 21, 71-83.

Michel, C. M., Murray, M. M., Lantz, G., Gonzalez, S., Spinelli, L., and Grave de Peralta, R. (2004b). EEG source imaging. Clin. Neurophysiol. 115, 2195-2222.

Mikuni, N., Nagamine, T., Ikeda, A., Terada, K., Taki, W., Kimura, J., et al. (1997). Simultaneous recording of epileptiform discharges by MEG and subdural electrodes in temporal lobe epilepsy. Neuroimage 5, 298-306.

Minassian, B. A., Otsubo, H., Weiss, S., Elliott, I., Rutka, J. T., and Snead, O. C. III (1999). Magnetoencephalographic localization in pediatric epilepsy surgery: comparison with invasive intracranial electroencephalography. Ann. Neurol. 46, 627-633.

Morioka, T., Nishio, S., Hisada, K., Shigeto, H., Yamamoto, T., Fujii, K., et al. (2000). Neuromagnetic assessment of epileptogenicity in cerebral arteriovenous malformation. Neurosurg. Rev. 23, 206-212.

Nakasato, N., Levesque, M. F., Barth, D. S., Baumgartner, C., Rogers, R. L., and Sutherling, W. W. (1994). Comparisons of MEG, EEG, and ECoG source localization in neocortical partial epilepsy in humans. Electroencephalogr. Clin. Neurophysiol. 91, 171-178.

Oishi, M., Otsubo, H., Kameyama, S., Morota, N., Masuda, H., Kitayama, M., et al. (2002). Epileptic spikes: magnetoencephalography versus simultaneous electrocorticography. Epilepsia 43, 1390-1395.

Otsubo, H., Sharma, R., Elliott, I. Holowka, S., Rutka, J. T., and Snead, O. C. III (1999). Confirmation of two magnetoencephalographic epileptic foci by invasive monitoring from subdural electrodes in an adolescent with right frontocentral epilepsy. Epilepsia 40, 608-613.

Otsubo, H., Steinlin, M., Hwang, P. A., Sharma, R., Jay, V., Becker, L. E., et al. (1997). Positive epileptiform discharges in children with neuronal migration disorders. Pediatr. Neurol. 16, 23-31.

Pascual-Marqui, R. D., Esslen, M., Kochi, K., and Lehmann, D. (2002). Functional imaging with lowresolution brain electromagnetic tomography (LORETA): a review. Methods Find Exp. Clin. Pharmacol. 24(Suppl. C), 91-95.

Plummer, C., Litewka, L., Farish, S., Harvey, A. S., and Cook, M. J. (2007). Clinical utility of current-generation dipole modelling of scalp EEG. Clin. Neurophysiol. 118, 2344-2361.

Rampp, S., and Stefan, H. (2007). Magnetoencephalography in presurgical epilepsy diagnosis. Expert Rev. Med. Devices 4, 335-347.

Ricci, G. B., Romani, G. L., Salustri, C., Pizzella, V., Torrioli, G., Buonomo, S., et al. (1987). Study of focal epilepsy by multichannel neuromagnetic measurements. Electroencephalogr. Clin. Neurophysiol. 66, 358-368.

Russell, G. S., Eriksen, K. J., Poolman, P., Luu, P., and Tucker, D. M. (2005). Geodesic photogrammetry for localizing sensor positions in dense-array EEG. Clin. Neurophysiol. 116, 1130-1140.

Stefan, H., Hummel, C., Scheler, G., Genow, A., Druschky, K., Tilz, C., et al. (2003). Magnetic brain source imaging of focal epileptic activity: a synopsis of 455 cases. Brain 126, 2396-2405.

Tucker, D. M., Holmes, M. D., Brown, M., and Vanhatalo, S. (2004). Improved spatial resolution of focal epileptiform discharges by dense array EEG recordings. Epilepsia 45 , 71-72. 
Vanrumste, B., Van Hoey, G., Van de Walle, R., D'Havé, M., Lemahieu, I., and Boon, P. (2000). Dipole location errors in electroencephalogram source analysis due to volume conductor model errors. Med. Biol. Eng. Comput. 38, 528-534.

Yamazaki, M., Tucker, D. M., Fujimoto, A., Yamazoe, T., Okanishi, T., Yokota, T., et al. (2012a). Comparison of dense array EEG with simultaneous intracranial EEG for interictal spike detection and localization. Epilepsy Res. 98, 166-173.

Yamazaki, M., Terrill, M., Fujimoto, A., Yamamoto, T., and Tucker, D. M. (2012b). Integrating dense array EEG in the presurgical evaluation of temporal lobe epilepsy. ISRN Neurol. 2012, 924081.

Conflict of Interest Statement: The authors declare that the research was conducted in the absence of any commercial or financial relationships that could be construed as a potential conflict of interest.

Received: 17 February 2013; paper pending published: 09 March 2013; accepted: 15 April 2013; published online: 13 May 2013.

Citation: Yamazaki M, Tucker DM, Terrill $M$, Fujimoto $A$ and Yamamoto $T$ (2013) Dense array EEG source estimation in neocortical epilepsy. Front. Neurol. 4:42. doi: 10.3389/fneur.2013.00042
This article was submitted to Frontier in Epilepsy, a specialty of Frontiers in Neurology.

Copyright (c) 2013 Yamazaki, Tucker, Terrill, Fujimoto and Yamamoto. This is an open-access article distributed under the terms of the Creative Commons Attribution License, which permits use, distribution and reproduction in other forums, provided the original authors and source are credited and subject to any copyright notices concerning any third-party graphics etc. 